\title{
A Joint Design Scheme for Identification and Control
}

\author{
Zhou Xiangrong and Y. S. Hung \\ Department of Electrical \& Electronic Engineering, University of Hong Kong, Hong Kong. \\ xrzhou@eee.hku.hk
}

\begin{abstract}
A design scheme for simultaneously solving for models and controllers based on a robust performance criterion is proposed. A suboptimal solution is provided.

\section{INTRODUCTION}

System identification must be considered in conjunction with its intended application. In agreement with robust control design paradigm, a popular approach is to identify not only a nominal model but also a worst case modeling error bound. Unfortunately the bound estimation is typically conservative and the effect of identification results to the subsequent robust control design is seldom considered in an integrated way. This consideration motivates an approach which may be called joint design of identification and control [3]. In general, a system identification criterion can be formulated as the minimization of control performance degradation due to the mismatch of a plant and a model. In [1], [4], system identification and control design are integrated into a single criterion, and an iterative solution for a model and a controller is proposed. Similar to [1],[4], we will propose a single design criterion for modeling and controller design. The solution is shown to have robust performance properties. Instead of an iterative scheme, we provide a suboptimal two step scheme to jointly solve for a model and a controller.
\end{abstract}

\section{A JOINT DESIGN CRITERION AND ITS PROPERTIES}

Suppose the plant $P$ is LTI and stable. Let $\widehat{P}$ denote a model for $P$ and $C$ a controller to be designed. Consider the control performance cost

$$
J \triangleq \|\left|\lambda(P-\widehat{P}) \frac{C}{1+\widehat{P} C}\right|+\left.\left|W_{1} \frac{1}{1+\widehat{P} C}\right|\right|_{\infty}
$$

where $W_{1}$ a stable transfer function weighting for performance and $\lambda$ a positive constant for specifying robustness. The form of $J$ is motivated by the robust performance cost for additive uncertainty given in [2]

$$
J_{r}(P, C)=\left\|\left|W_{2} \frac{C}{1+P C}\right|+\left|W_{1} \frac{1}{1+P C}\right|\right\|_{\infty}
$$

where $W_{2}$ is a stable transfer function defining the additive uncertainty bound. In fact, if $\widehat{P}=P-\frac{W_{2}}{\lambda}$, (1) turns into (2) as $\lambda \rightarrow \infty$. (1) is suitable as the performance cost for joint system identification and control design, because it combines controller design and data fitting into a single measure, where data fitting is taken into account by the term $(P-\widehat{P})$.

Given $\mathcal{P} \subseteq H^{\infty}$ and $\mathcal{C} \subseteq L^{\infty}$, define the admissible set of $\widehat{P}$ and $C$ for (1) as

$$
\mathcal{A} \triangleq\left\{(\widehat{P}, C) \mid \frac{1}{1+\widehat{P} C} \in H^{\infty}, \forall \widehat{P} \in \mathcal{P}, \forall C \in \mathcal{C}\right\}
$$

with no unstable zero-pole cancellation between $\widehat{P}$ and $C$. Let

$$
\left(\widehat{P}^{*}, C^{*}\right)=\arg J^{*}=\arg \min _{(\widehat{P}, C) \in \mathcal{A}} J(P, \widehat{P}, C)
$$

For comparison, we will also consider the minimal sensitivity problem:

$$
C^{o}=\arg J^{o}=\arg \min _{C \text { stablize } P}\left\|W_{1} \frac{1}{1+P C}\right\|_{\infty}
$$

The following theorem tells the relationship of (3) with (4) and with $\lambda$.

Theorem 1 Let $\mathcal{P}=H^{\infty}, \mathcal{C}=L^{\infty}$. (1) If $\lambda \geq J^{o}$, an optimal solution of (3) is $\left(P, C^{o}\right)$, furthermore $J^{*}=J^{o}$, $C^{*}=C^{o}$ uniquely. (2) If $\lambda<J^{o}$ and $\frac{k\left(1+P C^{o}\right)-1}{C^{o}}$ is stable for some $k>1$, then $J^{*}<J^{o}$.

Note that in the theorem $P$ is achievable by a model $\widehat{P}$ in the admissible set $\mathcal{P}$. If $P$ represents a nominal true plant which is not achievable as is usual in system identification, then the modelling error $P-\widehat{P}$ does not diminish. In this case, robustness is taken into account naturally in (1), which will be elaborated in the following.

We say that robust performance over a model set $\mathcal{X}$ is provided by a controller $C$ if for any $\bar{P} \in \mathcal{X}, C$ stabilizes $\bar{P}$ and $\left\|W_{1} \frac{1}{1+\bar{P} C}\right\|_{\infty}<1$. Define model sets

$\mathcal{Q} \triangleq\left\{\bar{P} \mid \bar{P}=\widehat{P}+\lambda(P-\widehat{P}) \Delta,\|\Delta\|_{\infty}<1\right\}$
$\mathcal{R} \triangleq\left\{\bar{P} \mid \bar{P}=P+(\lambda-1)(P-\widehat{P}) \Delta,\|\Delta\|_{\infty}<1\right\}$

Lemma $2 \lambda>1$ if and only if $\mathcal{R} \subseteq \mathcal{Q}$. 
Theorem 3 If $J(P, \widehat{P}, C)<1$ with $\lambda>1$, then $J_{r}(P, C)<1$ with $W_{2}=(\lambda-1)(P-\widehat{P})$.

By [2], a necessary and sufficient condition for robust performance over $\mathcal{Q}$ is $J(P, \widehat{P}, C)<1$. This together with Lemma 2 imply robust performance over $\mathcal{R}$. By [2], a necessary and sufficient condition for robust performance over $\mathcal{R}$ is $J_{r}(P, C)<1$ with $W_{2}=$ $(\lambda-1)(P-\widehat{P})$. This proves the theorem.

By our approach, while designing $\widehat{P}$ and $C$ to satisfy $J(P, \widehat{P}, C)<1$, we are actually designing $C$ so to provide robust performance over the model set $\mathcal{Q}$ centered at $\widehat{P}$. By the theorem, robust performance over $\mathcal{Q}$ guarantees robust performance over the model set $\mathcal{R}$ centered at $P$. Considering $P$ is the nominal true plant, this is what is desired. The novelty of this approach is that it is a $H^{\infty}$ style robust control design without the need to specify uncertainty bounds, which are hard to identify in system identification. Finally, it is observed that $\lambda$ can be used to adjust robustness levels and the degree of inclusion of $\mathcal{R} \subseteq \mathcal{Q}$.

\section{TWO STEP SOLUTION SCHEME AND AN EXAMPLE}

Since it is hard to solve $(3)$ or $J(P, \widehat{P}, C)<1$, we will replace $J$ by

$$
I(\widehat{P}, C) \triangleq\left\|\begin{array}{l}
\lambda(P-\widehat{P}) \frac{C}{1+\widehat{P} C} \\
W_{1} \frac{1}{1+\widehat{P} C}
\end{array}\right\|_{\infty}
$$

By the inequality $I \leq J \leq \sqrt{2} I$, Theorem 3 holds if $J<1$ is replaced with $I<\frac{1}{\sqrt{2}}$.

By the Youla parameterization, any stabilizing controller $C$ of $\widehat{P}$ can be written $C=\frac{Q}{1-\widehat{P} Q}, Q \in H^{\infty}$. We can therefore transform $I$ as

$$
I=\left\|\begin{array}{l}
\lambda(P-\widehat{P}) Q \\
W_{1}(1-\widehat{P} Q)
\end{array}\right\|_{\infty} \triangleq I_{1}(\widehat{P}, Q)
$$

Because $I_{1}$ is nonlinear in $(\widehat{P}, Q)$, this makes the synthesis task difficult. To overcome the difficulty, we will overbound $I_{1}$ with $I_{2}$ and $I_{3}$, defined as

$$
\begin{gathered}
I_{2}(Q, M) \triangleq\left\|\left(\begin{array}{l}
0 \\
W_{1}
\end{array}\right)-\left(\begin{array}{ll}
\lambda P & -\lambda \\
0 & W_{1}
\end{array}\right)\left(\begin{array}{c}
Q \\
M
\end{array}\right)\right\|_{\infty} \\
I_{3}(\widehat{P}, Q, M) \triangleq\left\|\left(\begin{array}{c}
-\lambda \\
W_{1}
\end{array}\right)(\widehat{P} Q-M)\right\|_{\infty}
\end{gathered}
$$

It can be shown by the triangle inequality that for any stable $\widehat{P}, Q$, and $M$,

$$
I_{1}(\widehat{P}, Q) \leq I_{2}(Q, M)+I_{3}(\widehat{P}, Q, M)
$$

Suppose a joint design criterion of modeling and control is: $\min _{\widehat{P}, Q} I_{1}(\widehat{P}, Q)$. For a suboptimal solution, a two step solution scheme is :
1. solve $(Q, M)$ from $\min _{Q, M} I_{2}(Q, M)$

2. solve $\widehat{P}$ from $\min _{\widehat{P}} I_{3}(\widehat{P}, Q, M)$.

With this scheme we have performed a design example for the plant

$$
P=\frac{z^{4}-1.2 z^{3}-0.3 z^{2}+0.156 z+0.0845}{z^{5}-1.25 z^{4}+0.458 z^{3}+0.028 z^{2}-0.049 z+0.0077}
$$

considered in [4]. Suppose the frequency response of $P$ is available at $\omega_{k}=k \frac{\pi}{100}, k=0,1, \ldots 99$. To facilitate computation, $\|\cdot\|_{\infty}$ is replaced by $\|\cdot\|_{2}$. Let $Q, M, \widehat{P}$ all have the form of $\varphi^{\tau} \theta$, where $\varphi$ consists of the basis $\left\{\frac{1}{z^{2}}\right\}$ and $\theta$ is a real parameter vector. The orders of $Q, M, \widehat{P}$ are taken to be $20,40,20$ respectively. With $\lambda=2$ and $W_{1}=\frac{z^{2}(z+0.2)}{(z+0.6)\left(z^{2}-0.648 z+0.36\right)}$, the sensitivity function $S=\frac{1}{1+P C}$ obtained using our approach compares favorably with that of [4] where joint identification and LQG control design is used. The Bode diagram of $S$ follows that of $W_{1}^{-1}$ nicely. With $\left\|I_{1}\right\|_{\infty}=1.0651$, we have that $J<1.0651 \sqrt{2}$. It follows from Theorem 3 that $W_{2}=0.3276(P-\widehat{P})$. Although the relative modeling error $\left|\frac{\hat{P}-P}{P}\right|$ is quite large ranging from $-5 d b$ to $10 \mathrm{db}$, but the control performance is good. We find that the choice of $\frac{W_{1}}{\lambda}$ is crucial for achieving the desired results. While keeping other conditions the same and letting $\lambda \rightarrow \infty$, it is interesting to note that $\widehat{P} \rightarrow P$ , $C \rightarrow$ a fixed controller and $W_{2} \rightarrow 0$.

\section{CONCLUSION}

We have proposed a joint identification and controller design scheme. The example shows that the scheme can work well. The effect of noise on the data is under investigation. We have used a linear parametrization and $\|\cdot\|_{2}$ in our example, but other kinds of parametrization and optimization with $\|\cdot\|_{\infty}$ can be considered.

\section{REFERENCES}

[1] D S Bayard, et al, 'A criterion for joint optimization of identification and robust control', IEEE Trans. Automat. Contr., vol 37, no.7, pp.986-991,1991

[2] John C Doyle, et al, 'Feedback control theory', Macmillan Publishing Company, New York.

[3] M. Gevers, 'Towards a joint design of identification and control', in Essays on control: perspectives in the theory and its applications, Birkhauser, pp.112149,1993

[4] Z Q Zang, et al,'Iterative weighted least-squares identification and weighted LQG control design', Automatica, Vol 31, No 11, pp1577-1594, 1995 